\title{
Inclusion theorems associated with a certain new family of asymptotically and statistically equivalent functions
}

\author{
H. M. Srivastavaa,b,*, Ekrem Savaş ${ }^{\mathrm{c}}$, Richard F. Patterson ${ }^{\mathrm{d}}$ \\ ${ }^{a}$ Department of Mathematics and Statistics, University of Victoria, Victoria, British Columbia V8W 3R4, Canada. \\ ${ }^{b}$ Department of Medical Research, China Medical University Hospital, China Medical University, Taichung 40402, Taiwan, Republic of \\ China. \\ ${ }^{c}$ Department of Mathematics, Istanbul Ticaret (Commerce) University, Sütlüce (Beyğlu), TR-34672 Üsküdar-Istanbul, Turkey. \\ ${ }^{d}$ Department of Mathematics and Statistics, University of North Florida, Jacksonville, Florida 32224, U.S.A.
}

Communicated by R. Saadati

\begin{abstract}
The aim of this paper is to introduce and investigate some new definitions which are interrelated to the notions of asymptotically $\mathrm{I}_{\lambda}$-statistical equivalence of multiple $\mathrm{L}$ and strongly $\mathrm{I}_{\lambda}$-asymptotic equivalence of multiple $\mathrm{L}$. Indeed, instead of sequences, the authors make use of two nonnegative real-valued Lebesgue measurable functions in the open interval $(1, \infty)$ and present a series of inclusion theorems associated with these new definitions. Furthermore, in connection with one of the main results which are proven in this paper, a closely-related open problem is posed for the interested reader.
\end{abstract}

Keywords: Ideals and filters, J-statistical convergence, $\mathrm{I}_{\lambda}$-statistical convergence, real-valued functions, asymptotically equivalent functions, $\lambda$-statistical convergence, de la Vallée Poussin method.

2010 MSC: 40H05, 40C05.

(C)2018 All rights reserved.

\section{Introduction and motivation}

The familiar and widely-investigated concept of convergence of a real sequence was extended to the notion of statistical convergence by Fast [8]. Schoenberg [38], on the other hand, gave fundamental properties of statistical convergence and also examined the idea of summability method. Šalát [27] showed that the set of bounded statistically convergent sequences is a closed subspace of the space of bounded sequences. Some other interesting works on statistically convergence can be found in (among others) $[3,4,9,10]$. Furthermore, for some recent developments on the subject of statistical convergence and related topics, the interested reader should refer (for example) to [1, 2, 12, 13, 21, 23, 39].

The relatively more general concept of J-convergence was introduced by Kostyrko et al. [14] in a metric space as a generalized form of the concept of statistical convergence and it is based upon the notion of an

\footnotetext{
*Corresponding author

Email addresses: harimsri@math.uvic.ca (H. M. Srivastava), ekremsavas@yahoo.com (Ekrem Savaş), rpatters@unf.edu (Richard F. Patterson)
}

doi: $10.22436 /$ jnsa.011.10.05

Received: 2017-08-13 Revised: 2018-01-06 Accepted: 2018-01-11 
ideal of the subset of the set $\mathbb{N}$ of positive integers. Later on it was further studied by Dems [7] and by Das et al. [6] (see also [16]). Further investigations in this direction and other applications of ideals can be found in the earlier works [20, 22, 28, 30-32, 34, 36, 37], and many other authors. Many other interesting applications of ideals can also be found in $[5,26]$.

Marouf [18] presented definitions for asymptotically equivalent sequences and asymptotically regular matrices. Li [17], on the other hand, presented and studied asymptotic equivalence of sequences and summability. Subsequently, Patterson [25] extended these concepts by presenting asymptotically statistical equivalence analogues of these definitions and natural regularity conditions for nonnegative summability matrices. Recently, Savaş and Basarir [35] introduced and investigated the $(\sigma, \lambda)$ - asymptotically statistical equivalent sequences. Six years later, the notion of asymptotically $\mathcal{J} \geqslant$-statistical equivalent sequences was studied by Gumus and Savas [11] (see also the work by Kumar and Sharma [15]).

Let $\lambda=\left(\lambda_{p}\right)$ be a non-decreasing sequence of positive numbers tending to $\infty$ such that

$$
\lambda_{1}:=1, \quad \lambda_{p+1} \leqq \lambda_{p}+1 \quad(p \in \mathbb{N}) \quad \text { and } \quad \lim _{p \rightarrow \infty} \lambda_{p}=\infty
$$

The set of all such sequences as the sequence $\lambda$ will be denoted by $\Delta$.

Mursaleen [19] studied the notion of convergence with respect to $\lambda$-statistical convergence. In this paper, he presented a series of critical results, beginning with the following definition.

Definition 1.1 ([19]). A sequence $x=\left(x_{k}\right)$ is said to be $\lambda$-statistically convergent or $S_{\lambda}$-convergent to the number $L$ if, for each $\varepsilon>0$,

$$
\lim _{p \rightarrow \infty}\left\{\frac{1}{\lambda_{p}}\left|\left\{k \in I_{p}:\left|x_{k}-L\right| \geqq \varepsilon\right\}\right|\right\}=0 .
$$

In this case, we write

$$
S_{\lambda} \lim _{k \rightarrow \infty} x_{k}=L \quad \text { or } \quad x_{k} \rightarrow L\left(S_{\lambda}\right)
$$

and we denote by $S_{\lambda}$ the set of all $\lambda$-statistically convergent sequences.

Very recently, Savaş [33] studied the generalized statistically convergent functions by using ideals. On the other hand, Savaş [29] presented a new approach to two well-known summability methods by using ideals and functions. The main object of this paper is to introduce the concept of asymptotically $\mathcal{J}_{\lambda}$-statistically equivalent for nonnegative real-valued functions and to investigate some of its main convergence properties.

\section{A set of preliminary definitions and associated properties}

A family $\mathcal{J} \subset 2^{Y}$ of subsets of a nonempty set $Y$ is said to be an ideal in $Y$ if

(i) the empty set $\emptyset \in \mathcal{J}$;

(ii) the conditions $A, B \in \mathcal{J}$ imply that $A \cup B \in \mathcal{J}$;

(iii) the conditions $A \in \mathcal{J}$ and $B \subset A$ imply that $B \in \mathcal{J}$. Furthermore, an admissible ideal $\mathcal{J}$ of $Y$ satisfies $\{x\} \in \mathcal{J}$ for each $x \in Y$. If $\mathcal{J}$ is an ideal in $Y$, then the following collection:

$$
\mathrm{F}(\mathcal{J})=\left\{M \subset Y \quad \text { and } \quad M^{\mathrm{c}} \in \mathcal{J}\right\}
$$

forms a filter in $\mathrm{Y}$ which is called the filter associated with $\mathcal{J}$.

Let $\mathcal{J} \subset 2^{\mathbb{N}}$ be a nontrivial ideal in $\mathbb{N}$. The sequence $\left(x_{k}\right)$ is said to be $\mathcal{J}$-convergent to $x$ if, for each $\varepsilon>0$, the set $\mathcal{A}(\varepsilon)$ given by

$$
A(\varepsilon)=\left\{k \in \mathbb{N}:\left|x_{k}-x\right| \geqq \varepsilon\right\}
$$

belongs to J (see [14]).

Let us begin our present investigation with the following definitions and preliminaries. 
Definition 2.1 ([18]). Two nonnegative sequences $x=\left(x_{k}\right)$ and $y=\left(y_{k}\right)$ are said to be asymptotically equivalent if

$$
\lim _{k \rightarrow \infty}\left\{\frac{x_{k}}{y_{k}}\right\}=1
$$

We denote this asymptotic equivalence by $x \sim y$.

Definition 2.2 ([10]). The sequence $x=\left(x_{k}\right)$ is said to be statistically convergent to the number $L$ if, for each $\varepsilon>0$,

$$
\lim _{n \rightarrow \infty}\left\{\frac{1}{n}\left|\left\{k \leqq n:\left|x_{k}-L\right| \geqq \varepsilon\right\}\right|\right\}=0 .
$$

We denote this statistical convergence by stat $\lim x_{k}=\mathrm{L}$.

Patterson [25] presented the following definition.

Definition 2.3 ([25]). Two nonnegative sequences $x=\left(x_{k}\right)$ and $y=\left(y_{k}\right)$ are said to be asymptotically statistically equivalent of multiple L provided that, for each $\varepsilon>0$,

$$
\lim _{n \rightarrow \infty}\left\{\frac{1}{n}\left|\left\{k \leqq n:\left|\frac{x_{k}}{y_{k}}-L\right| \geqq \varepsilon\right\}\right|\right\}=0 .
$$

We denote this equivalence by $x \stackrel{S_{L}}{\sim} y$; it is simply asymptotically statistically equivalent if $L=1$.

The generalized de la Vallée Poussin mean is defined as follows:

$$
t_{p}(x)=\frac{1}{\lambda_{p}} \sum_{k \in \mathcal{J}_{p}} x_{k}
$$

where

$$
\mathrm{I}_{\mathrm{p}}=\left[\mathrm{p}-\lambda_{\mathrm{p}}+1, \mathrm{p}\right] \quad(\mathrm{p} \in \mathbb{N}) .
$$

A sequence $x=\left(x_{k}\right)$ is said to be $[V, \lambda]$-summable to a number $L$ if $t_{p}(x) \rightarrow L$ and $n \rightarrow \infty$. We write

$$
[V, \lambda]=\lim _{p \rightarrow \infty}\left\{\frac{1}{\lambda_{p}} \sum_{k \in \mathcal{J}_{p}}\left|x_{k}-L\right|\right\}=0 \text { for some } L
$$

for the set of sequences that are strongly summable by the de la Vallée Poussin method. In the special case when $\lambda_{p}=p(p \in \mathbb{N})$, the set $[V, \lambda]$ reduces to the set in the $[C, 1]$-summability which is defined as follows:

$$
[C, 1]=\lim _{p \rightarrow \infty}\left\{\frac{1}{p} \sum_{k=1}^{p}\left|x_{k}-L\right|\right\}=0 \text { for some L. }
$$

We are now ready to give the following definitions.

Definition 2.4. Let $\lambda \in \Lambda$ and let $\chi(\xi)$ be a nonnegative real-valued function which is Lebesgue measurable in the open interval $(1, \infty)$ if

$$
\text { J- } \lim _{p \rightarrow \infty}\left\{\frac{1}{\lambda_{p}} \int_{p-\lambda_{p}+1}^{p}|x(\xi)-L| d \xi\right\}=0 .
$$

We say that the function $x(\xi)$ is $[\mathrm{V}, \lambda](\mathcal{J})$-summable to L. If

$$
\mathcal{J}=\mathcal{J}_{\text {fin }}=\{A \subseteq \mathbb{N}: A \text { is a finite subset }\},
$$


then the $[\mathrm{V}, \lambda](\mathcal{J})$-summability becomes the $[\mathrm{V}, \lambda]$-summability, which is defined as follows (see [24]):

$$
\lim _{p \rightarrow \infty}\left\{\frac{1}{\lambda_{p}} \int_{p-\lambda_{p}+1}^{p}|x(\xi)-L| d \xi\right\}=0 .
$$

Definition 2.5. A nonnegative real-valued function $x(\xi)$ is said to be $\mathcal{J}_{\lambda}$-statistically convergent or $S_{\lambda}(\mathcal{J})$ convergent to $L$ if, for every $\epsilon>0$ and $\delta>0$,

$$
\left\{p \in \mathbb{N}: \frac{1}{\lambda_{p}}\left|\left\{\xi \in I_{p}:|\chi(\xi)-\mathrm{L}| \geqq \epsilon\right\}\right| \geqq \delta\right\} \in \mathcal{J} .
$$

In this case, we write

$$
\mathrm{S}_{\lambda}(\mathcal{J})-\lim x(\xi)=\mathrm{L} \quad \text { or } \quad x(\xi) \rightarrow \mathrm{L}\left(\mathrm{S}_{\lambda}(\mathcal{J})\right)
$$

For $\mathcal{J}=\mathcal{J}_{\text {fin }}$, the $S_{\lambda}(\mathcal{J})$-convergence again coincides with the $\lambda$-statistical convergence (see [24]).

Following the above definitions, we introduce several new definitions in Section 3 below, which are related to the notions of asymptotically $\mathcal{J}_{\lambda}$-statistically equivalent of multiple $L$ and strongly $\mathcal{J}_{\lambda^{-}}$ asymptotically equivalent of multiple $L$ for nonnegative real-valued functions $x(\xi)$ and $y(\xi)$.

\section{The main definitions}

The above results lead us naturally to the following new definitions.

Definition 3.1. Let $\lambda \in \Lambda$. Also let $x(\xi)$ and $y(t)$ be two nonnegative real-valued Lebesgue measurable functions in the open interval $(1, \infty)$. We say that the sequences $x(\xi)$ and $y(\xi)$ are $\lambda$-asymptotically statistically equivalent of multiple $L$ if, for each $\epsilon>0$,

$$
\left\{p \in \mathbb{N}: \frac{1}{\lambda_{p}}\left|\left\{\xi \in I_{p}:\left|\frac{x(\xi)}{y(\xi)}-L\right| \geqq \epsilon\right\}\right|\right\}=0 .
$$

We denote this equivalence by

$$
\left.x(\xi) \stackrel{S_{\lambda}^{L}}{\sim} y(\xi)\right)
$$

and call it simply asymptotically statistically equivalent if $L=1$.

Definition 3.2. Let $\lambda \in \Lambda$ and let $\mathcal{J}$ be an admissible ideal in $\mathbb{N}$ and $x(\xi)$ and $y(\xi)$ be two nonnegative real-valued Lebesgue measurable functions in the open interval $(1, \infty)$. We say that the sequences $x(\xi)$ and $y(\xi)$ are strongly $J_{\lambda}$-asymptotically equivalent of multiple $L$ if, for each $\varepsilon>0$,

$$
\text { J- } \lim _{p \rightarrow \infty}\left\{\frac{1}{\lambda_{p}} \int_{p-\lambda_{p}+1}^{p}\left|\frac{x(\xi)}{y(\xi)}-L\right| d \xi\right\}=0 .
$$

Notationally, we write this as follows:

$$
x(\xi) \stackrel{V_{\lambda}^{L}(\mathcal{J})}{\sim} y(\xi)
$$

and call it simply asymptotically statistical equivalence if $L=1$.

If

$$
\mathcal{J}=\mathcal{J}_{\text {fin }}=\{A \subseteq \mathbb{N}: A \text { is a finite subset }\},
$$

the strongly $\mathcal{J}_{\lambda}$-asymptotically equivalent reduces to strongly $\lambda$-asymptotically equivalent, defined as follows:

$$
\lim _{p \rightarrow \infty}\left\{\frac{1}{\lambda_{p}} \int_{p-\lambda_{p}+1}^{p}\left|\frac{x(\xi)}{y(\xi)}-L\right| d \xi\right\}=0
$$


Definition 3.3. Let $x(\xi)$ and $y(\xi)$ be two nonnegative real-valued Lebesgue measurable functions in the open interval $(1, \infty)$ and $\mathcal{J}$ be an admissible ideal in $\mathbb{N}$. We say that the functions $x(\xi)$ and $y(\xi)$ are asymptotically $\mathcal{J}_{\lambda}$-statistically equivalent of multiple $L$ if, for every $\epsilon>0$ and $\delta>0$,

$$
\left\{p \in \mathbb{N}: \frac{1}{p}\left|\left\{k \leqq p:\left|\frac{x(\xi)}{y(\xi)}-L\right| \geqq \varepsilon\right\}\right| \geqq \delta\right\} \in \mathcal{J}
$$

In this case, we write

$$
x(\xi) \stackrel{S^{L}(\mathcal{J})}{\sim} y(\xi)
$$

By combining Definitions 2.1 and 2.5, we have the following new definition.

Definition 3.4. Let $\lambda \in \Lambda$ and $\mathcal{J}$ be an admissible ideal in $\mathbb{N}$. Also let $x(\xi)$ and $y(\xi)$ be two nonnegative real-valued Lebesgue measurable functions in the open interval $(1, \infty)$. We say that the functions $x(\xi)$ and $y(\xi)$ are $\mathcal{J}_{\lambda}$-asymptotically statistically equivalent of multiple $L$ if, for every $\epsilon>0$ and $\delta>0$,

$$
\left\{\mathrm{p} \in \mathbb{N}: \frac{1}{\lambda_{\mathrm{p}}}\left|\left\{\xi \in \mathrm{I}_{\mathfrak{p}}:\left|\frac{x(\xi)}{\mathrm{y}(\xi)}-\mathrm{L}\right| \geqq \epsilon\right\}\right| \geqq \delta\right\} \in \mathcal{J} .
$$

We denote this equivalence by

$$
x(\xi) \stackrel{S_{\lambda}^{\mathrm{L}}(\mathcal{J})}{\sim} y(\xi)
$$

and call it simply asymptotically statistically equivalent if $L=1$.

For $\mathcal{J}=\mathcal{J}_{\text {fin }}$, the $\mathcal{J}_{\lambda}$-asymptotically statistically equivalence reduces to the $\lambda$-asymptotically statistically equivalence which is already given in Definition 3.1.

\section{The main inclusion theorems}

Theorem 4.1. Let $\lambda=\left(\lambda_{n}\right) \in \Delta$. Also let $x(\xi)$ and $y(\xi)$ be real-valued functions which are Lebesgue measurable in the open interval $(1, \infty)$. Then each of the following assertions holds true.

(i) If $x(\xi) \stackrel{V_{\lambda}^{\mathrm{L}}(\mathcal{J})}{\sim} \mathrm{y}(\xi)$, then $x(\xi) \stackrel{S_{\lambda}^{\mathrm{L}}(\mathcal{J})}{\sim} \mathrm{y}(\xi)$ and the inclusion is proper for every ideal $\mathcal{J}$.

(ii) If the functions $x(\xi)$ and $y(\xi)$ are bounded and $x(\xi) \stackrel{S_{\lambda}^{L}(\mathcal{J})}{\sim} y(\xi)$, then $x(\xi) \stackrel{V_{\lambda}^{L}(\mathcal{J})}{\sim} y(\xi)$.

Proof.

(i) Let $\epsilon>0$ and

$$
x(\xi) \stackrel{V_{\lambda}^{L}(\mathcal{J})}{\sim} y(\xi)
$$

We thus find that

$$
\int_{\xi \in I_{p}}\left|\frac{x(\xi)}{y(\xi)}-L\right| d \xi \geqq \int_{\xi \in I_{p}}\left(\left|\frac{x(\xi)}{y(\xi)}-L\right|>\varepsilon\right)\left|\frac{x(\xi)}{y(\xi)}-L\right| d \xi \geqq \epsilon \cdot\left|\left\{\xi \in I_{p}:\left|\frac{x(\xi)}{y(\xi)}-L\right| \geqq \epsilon\right\}\right| .
$$

So, for a given $\delta>0$, we have

$$
\frac{1}{\lambda_{\mathrm{p}}}\left|\left\{\xi \in \mathrm{I}_{\mathrm{p}}:\left|\frac{x(\xi)}{\mathrm{y}(\xi)}-\mathrm{L}\right| \geqq \epsilon\right\}\right| \geqq \delta \Longrightarrow \frac{1}{\lambda_{\mathrm{p}}} \int_{\xi \in \mathrm{I}_{\mathrm{p}}}\left|\frac{x(\xi)}{\mathrm{y}(\xi)}-\mathrm{L}\right| \geqq \epsilon \delta,
$$

that is,

$$
\left\{p \in \mathbb{N}: \frac{1}{\lambda_{\mathfrak{p}}}\left|\left\{\xi \in \mathrm{I}_{\mathfrak{p}}:\left|\frac{x(\xi)}{\mathrm{y}(\xi)}-\mathrm{L}\right| \geqq \epsilon\right\}\right| \geqq \delta\right\} \subset\left\{p \in \mathbb{N}: \frac{1}{\lambda_{\mathfrak{p}}}\left(\int_{\xi \in \mathrm{I}_{\mathfrak{p}}}\left|\frac{x(\xi)}{\mathrm{y}(\xi)}-\mathrm{L}\right| \geqq \epsilon\right) \geqq \epsilon \delta\right\} .
$$


Since the right-hand side of (4.1) belongs to J, we conclude that left-hand side belongs to J.

In order to show that

$$
x(\xi) \stackrel{S_{\lambda}^{\mathrm{L}}(\mathcal{J})}{\sim} y(\xi) \varsubsetneqq x(\xi) \stackrel{V_{\lambda}^{\mathrm{L}}(\mathcal{J})}{\sim} y(\xi),
$$

we take a fixed $A \in \mathcal{J}$. Define a function $x(\xi)$ by

$$
x(\xi)= \begin{cases}\xi, & \left(p-\left[\sqrt{\lambda_{p}}\right]+1 \leqq \xi \leqq p ; p \notin A\right) \\ \xi, & \left(p-\lambda_{p}+1 \leqq \xi \leqq p ; p \in A\right) \\ \theta, & \text { (otherwise }),\end{cases}
$$

and let $y(\xi)=1$. Then, for every $\epsilon(0<\epsilon<1)$, we have

$$
\frac{1}{\lambda_{p}}\left|\left\{\xi \in I_{p}:\left|\frac{x(\xi)}{y(\xi)}-L\right| \geqq \epsilon\right\}\right|=\frac{\sqrt{\lambda_{p}}}{\lambda_{p}} \rightarrow 0
$$

as $n \rightarrow \infty$ and $n \notin A$. Therefore, for every $\delta>0$, we find that

$$
\left\{p \in \mathbb{N}: \frac{1}{\lambda_{p}}\left|\left\{\xi \in I_{p}:\left|\frac{x(\xi)}{y(\xi)}-L\right| \geqq \epsilon\right\}\right| \geqq \delta\right\} \subset A \cup\{1,2,3, \ldots, m\}
$$

for some $m \in \mathbb{N}$. Since $\mathcal{J}$ is admissible, it follows that it is asymptotically $\mathcal{J}_{\lambda}$-statistically equivalent of multiple L. Thus, obviously, we get

$$
\frac{1}{\lambda_{p}} \int_{\xi \in I_{p}}\left|\frac{x(\xi)}{y(\xi)}-\mathrm{L}\right| \rightarrow \infty \quad(p \rightarrow \infty)
$$

that is, it is not strongly $\mathcal{J}_{\lambda}$-asymptotically equivalent of multiple $L$. If $A \in \mathcal{J}$ is infinite, then it is not $\mathcal{J}_{\lambda}$-asymptotically statistically equivalent of multiple $\mathrm{L}$.

(ii) Suppose that $x(\xi) \stackrel{S_{\lambda}^{L}(\mathcal{J})}{\sim} y(\xi)$ and that the following quotient:

$$
\frac{x(t)}{y(t)}
$$

is bounded by $M$. Let $\epsilon>0$ be given. Now

$$
\begin{aligned}
& \frac{1}{\lambda_{\mathfrak{p}}} \int_{\xi \in \mathrm{I}_{\mathfrak{p}}}\left|\frac{x(\xi)}{\mathrm{y}(\xi)}-\mathrm{L}\right|=\frac{1}{\lambda_{\mathfrak{p}}} \int_{\xi \in \mathrm{I}_{\mathfrak{p}}\left(\left|\frac{x(\xi)}{y(\xi)}-\mathrm{L}\right| \geq \varepsilon\right)}\left|\frac{x(\xi)}{y(\xi)}-\mathrm{L}\right|+\frac{1}{\lambda_{\mathfrak{p}}} \int_{\xi \in \mathrm{I}_{\mathfrak{p}}\left(\left|\frac{x(\xi)}{y(\xi)}-\mathrm{L}\right|<\varepsilon\right)}|x(\xi)-\mathrm{L}| \\
& \leqq \frac{M}{\lambda_{p}}\left\{\xi \in I_{p}:\left|\frac{x(\xi)}{y(\xi)}-L\right| \geqq \epsilon\right\}+\epsilon .
\end{aligned}
$$

We note that

$$
\left\{p \in N: \frac{1}{\lambda_{p}}\left|\left\{\xi \in I_{p}:\left|\frac{x(\xi)}{y(\xi)}-L\right| \geqq \epsilon\right\}\right| \geqq \frac{\epsilon}{M}\right\}=: A(\epsilon) \in \mathcal{J} .
$$

Thus, if $p \in[A(\epsilon)]^{c}$, we get

$$
\frac{1}{\lambda_{\mathrm{p}}} \sum_{\xi \in \mathrm{I}_{\mathrm{p}}}\left|\frac{x(\xi)}{\mathrm{y}(\xi)}-\mathrm{L}\right|<2 \epsilon .
$$

Hence, obviously, we have

$$
\left\{p \in \mathbb{N}: \frac{1}{\lambda_{p}} \sum_{\xi \in I_{p}}\left|\frac{x(\xi)}{y(\xi)}-L\right| \geqq 2 \epsilon\right\} \subset A(\epsilon)
$$


and so it belongs to J. This shows that

$$
x(\xi) \stackrel{V_{\lambda}^{L}(\mathcal{J})}{\sim} y(\xi)
$$

The proof of Theorem 4.1 is evidently completed.

Theorem 4.2. If

$$
\liminf _{p \rightarrow \infty}\left\{\frac{\lambda_{p}}{p}\right\}>0
$$

then

$$
x(\xi) \stackrel{S^{\mathrm{L}}(\mathcal{J})}{\sim} y(\xi) \subset x(\xi) \stackrel{S_{\lambda}^{\mathrm{L}}(\mathcal{J})}{\sim} y(\xi)
$$

Proof. For each $€>0$, we write

$$
\frac{1}{p}\left|\left\{\xi \leqq p:\left|\frac{x(\xi)}{y(\xi)}-L\right| \geqq \epsilon\right\}\right| \geqq \frac{1}{p}\left|\left\{\xi \in I_{p}:\left|\frac{x(\xi)}{y(\xi)}-L\right| \geqq \epsilon\right\}\right| \geqq \frac{\lambda_{p}}{p} \frac{1}{\lambda_{p}}\left|\left\{\xi \in I_{p}:\left|\frac{x(\xi)}{y(\xi)}-L\right| \geqq \epsilon\right\}\right| .
$$

Hence, if

$$
\liminf _{p \rightarrow \infty}\left\{\frac{\lambda_{p}}{p}\right\}=a
$$

then (by definition) the following set:

$$
\left\{p \in N: \frac{\lambda_{p}}{p}<\frac{a}{2}\right\}
$$

is finite. Thus, for $\delta>0$, we find that

$$
\begin{aligned}
\left\{p \in \mathbb{N}: \frac{1}{\lambda_{p}}\left|\left\{\xi \in I_{p}:\left|\frac{x(\xi)}{y(\xi)}-L\right| \geqq \epsilon\right\}\right| \geqq \delta\right\} \\
\quad \subset\left\{p \in N: \frac{1}{p}\left|\left\{\xi \in I_{p}:\left|\frac{x(\xi)}{y(\xi)}-L\right| \geqq \epsilon\right\}\right| \geqq \frac{a}{2} \delta\right\} \cup\left\{p \in N: \frac{\lambda_{p}}{p}<\frac{a}{2}\right\} .
\end{aligned}
$$

Since $\mathcal{J}$ is admissible, the set on the right-hand side belongs to J. Clearly, we have completed the proof of Theorem 4.2.

Theorem 4.3. Let the parameter $\lambda \in \Delta$ be such that

$$
\lim _{p \rightarrow \infty}\left\{\frac{\lambda_{p}}{p}\right\}=1
$$

Then

$$
x(\xi) \stackrel{S_{\lambda}^{\mathrm{L}}(\mathcal{J})}{\sim} y(\xi) \subset x(\xi) \stackrel{S^{\mathrm{L}}(\mathcal{J})}{\sim} y(\xi) .
$$

Proof. Let $\delta>0$ be given. Since, by hypothesis,

$$
\lim _{p \rightarrow \infty}\left\{\frac{\lambda_{p}}{p}\right\}=1,
$$

we can choose $m \in \mathbb{N}$ such that

$$
\left|\frac{\lambda_{p}}{p}-1\right|<\frac{\delta}{2} \quad(p \geqq m) .
$$

We now observe that, for $\varepsilon>0$,

$$
\frac{1}{p}\left|\left\{\xi \leqq p:\left|\frac{x(\xi)}{y(\xi)}-L\right| \geqq \epsilon\right\}\right|=\frac{1}{p}\left|\left\{\xi \leqq p-\lambda_{p}:\left|\frac{x(\xi)}{y(\xi)}-L\right| \geqq \epsilon\right\}\right|+\frac{1}{p}\left|\left\{\xi \in I_{p}:\left|\frac{x(\xi)}{y(\xi)}-L\right| \geqq \epsilon\right\}\right|
$$




$$
\begin{aligned}
& \leqq \frac{p-\lambda_{p}}{p}+\frac{1}{p}\left|\left\{\xi \in I_{p}:\left|\frac{x(\xi)}{y(\xi)}-L\right| \geqq \epsilon\right\}\right| \\
& \leqq 1-\left(1-\frac{\delta}{2}\right)+\frac{1}{p}\left|\left\{t \in I_{p}:\left|\frac{x(\xi)}{y(\xi)}-L\right| \geqq \epsilon\right\}\right| \\
& =\frac{\delta}{2}+\frac{1}{p}\left|\left\{\xi \in I_{p}:|x(\xi)-L| \geqq \epsilon\right\}\right|
\end{aligned}
$$

for all $n \geqq m$. Hence, obviously, we can write

$$
\left\{p \in \mathbb{N}: \frac{1}{p}|\{\xi \leqq p:|x(\xi)-L| \geqq \epsilon\}| \geqq \delta\right\} \subset\left\{p \in \mathbb{N}: \frac{1}{p}\left|\left\{\xi \in I_{p}:|x(\xi)-L| \geqq \epsilon\right\}\right| \geqq \frac{\delta}{2}\right\} \cup\{1,2,3, \ldots, m\} .
$$

This shows that

$$
x(\xi) \stackrel{S^{L}(\mathcal{J})}{\sim} y(\xi)
$$

The proof of Theorem 4.3 is thus completed.

Remark 4.4 (An open problem). We do not yet know whether the condition in Theorem 4.3 is necessary. Therefore, we choose to leave it as an open problem for the interested reader.

Finally, we complete this paper by presenting the following result.

Theorem 4.5. Let $\lambda=\left(\lambda_{p}\right)$ and $\mu=\left(\mu_{p}\right)$ be two sequences in $\triangle$ such that

$$
\lambda_{\mathrm{p}} \leqq \mu_{\mathrm{p}} \quad(p \in \mathbb{N}) .
$$

Then each of the following assertions holds true.

(i) If

$$
\liminf _{p \rightarrow \infty}\left\{\frac{\lambda_{p}}{\mu_{p}}\right\}>0
$$

(ii) If

$$
\text { then } x(\xi) \stackrel{S_{\mu}^{L}(\mathcal{J})}{\sim} y(\xi) \subset x(\xi) \stackrel{S_{\lambda}^{L}(\mathcal{J})}{\sim} y(\xi) .
$$

$$
\lim _{p \rightarrow \infty}\left\{\frac{\mu_{p}}{\lambda_{p}}\right\}=1
$$

$$
\text { then } x(\xi) \stackrel{S_{\lambda}^{L}(\mathcal{J})}{\sim} y(\xi) \subset x(\xi) \stackrel{S_{\mu}^{L}(\mathcal{J})}{\sim} y(\xi) .
$$

Proof.

(i) Suppose that $\lambda_{p} \leqq \mu_{p}$ for all $p \in \mathbb{N}$ and that the condition (4.2) is satisfied. Then, for $\varepsilon>0$, we have

$$
\left\{\xi \in J_{p}:\left|\frac{x(\xi)}{y(\xi)}-L\right| \geqq \epsilon\right\} \supseteq\left\{\xi \in I_{n}:\left|\frac{x(\xi)}{y(\xi)}-L\right| \geqq \epsilon\right\} .
$$

Therefore, we can write

$$
\frac{1}{\mu_{p}}\left|\left\{\xi \in J_{p}:\left|\frac{x(\xi)}{y(\xi)}-L\right| \geqq \epsilon\right\}\right| \geqq \frac{\lambda_{p}}{\mu_{p}} \frac{1}{\lambda_{p}}\left|\left\{\xi \in I_{p}:\left|\frac{x(\xi)}{y(\xi)}-L\right| \geqq \epsilon\right\}\right| .
$$

Thus, for all $p \in \mathbb{N}$, we find that

$$
\left\{p \in \mathbb{N}: \frac{1}{\lambda_{p}}\left|\left\{\xi \in I_{p}:\left|\frac{x(\xi)}{y(\xi)}-L\right| \geqq \epsilon\right\}\right| \geqq \delta\right\} \subseteq\left\{p \in \mathbb{N}: \frac{1}{\mu_{p}}\left|\left\{\xi \in J_{p}:\left|\frac{x(\xi)}{y(\xi)}-L\right| \geqq \epsilon\right\}\right| \geqq \delta \frac{\lambda_{p}}{\mu_{p}}\right\} \in \mathcal{J} .
$$

Hence we get

$$
x(\xi) \stackrel{S_{\mu}^{L}(\mathcal{I})}{\sim} y(\xi) \subset x(\xi) \stackrel{S_{\lambda}^{L}(\mathcal{J})}{\sim} y(\xi)
$$


(ii) Let $x(\xi) \stackrel{S_{\lambda}^{L}(\mathcal{J})}{\sim} y(\xi)$. Suppose also that the condition (4.3) is satisfied. Since $I_{n} \subset J_{n}$, for $\varepsilon>0$, we may write

$$
\begin{aligned}
\frac{1}{\mu_{p}}\left|\left\{\xi \in J_{p}:\left|\frac{x(\xi)}{y(\xi)}-\mathrm{L}\right| \geqq \epsilon\right\}\right| & =\frac{1}{\mu_{p}}\left|\left\{p-\mu_{p}+1<t \leqq p-\lambda_{p}:\left|\frac{x(\xi)}{y(\xi)}-\mathrm{L}\right|\right\}\right| \\
& +\frac{1}{\mu_{p}}\left|\left\{\xi \in I_{p}:\left|\frac{x(\xi)}{y(\xi)}-\mathrm{L}\right| \geqq \epsilon\right\}\right| \\
\leqq & \frac{\mu_{p}-\lambda_{p}}{\mu_{p}}+\frac{1}{\lambda_{p}}\left|\left\{\xi \in I_{p}:\left|\frac{x(\xi)}{y(\xi)}-\mathrm{L}\right| \geqq \epsilon\right\}\right| \\
\leqq & \left(\frac{\mu_{p}-\lambda_{p}}{\lambda_{p}}\right)+\frac{1}{\lambda_{p}}\left|\left\{\xi \in I_{p}:\left|\frac{x(\xi)}{y(\xi)}-L\right| \geqq \epsilon\right\}\right| \\
& \left(\frac{\mu_{p}}{\lambda_{p}}-1\right)+\frac{1}{\lambda_{p}}\left|\left\{\xi \in I_{p}:\left|\frac{x(\xi)}{y(\xi)}-L\right| \geqq \epsilon\right\}\right|
\end{aligned}
$$

for all $p \in \mathbb{N}$. Hence we have

$$
\left\{p \in \mathbb{N}: \frac{1}{\mu_{p}}\left|\left\{\xi \in J_{p}:\left|\frac{x(\xi)}{y(\xi)}-L\right| \geqq \epsilon\right\}\right| \geqq \delta\right\} \subseteq\left\{p \in \mathbb{N}: \frac{1}{\lambda_{p}}\left|\left\{\xi \in I_{p}:\left|\frac{x(\xi)}{y(\xi)}-L\right| \geqq \epsilon\right\}\right| \geqq \delta\right\} \in \mathcal{J} .
$$

This implies that

$$
x(\xi) \stackrel{S_{\lambda}^{L}(\mathcal{J})}{\sim} y(\xi) \subset x(t) \stackrel{S_{\mu}^{L}(\mathcal{J})}{\sim} y(\xi) .
$$

We thus have completed the proof of Theorem 4.5.

Many other interesting corollaries and consequences of the various definitions and results, which we have presented in this investigation, are potentially fruitful for further studies on the subject of statistical convergence and related areas.

\section{References}

[1] M. Aldhaifallah, K. S. Nisar, H. M. Srivastava, M. Mursaleen, Statistical $\Lambda$-convergence in probabilistic normed spaces, J. Funct. Spaces, 2017 (2017), 7 pages. 1

[2] N. L. Braha, V. Loku, H. M. Srivastava, $\wedge^{2}$-Weighted statistical convergence and Korovkin and Voronovskaya type theorems, Appl. Math. Comput., 266 (2015), 675-686. 1

[3] H. Cakalli, A study on statistical convergence, Funct. Anal. Approx. Comput., 1 (2009), 19-24. 1

[4] J. Connor, E. Savaş, Lacunary statistical and sliding window convergence for measurable functions, Acta Math. Hungar., 145 (2015), 416-432. 1

[5] P. Das, P. Kostyrko, W. Wilczynski, P. Malik, I- and I*-convergence of double sequences, Math. Slovaca, 58 (2008), 605-620. 1

[6] P. Das, E. Savaş, S. K. Ghosal, On generalizations of certain summability methods using ideals, Appl. Math. Lett., 24 (2011), 1509-1514. 1

[7] K. Dems, On I-Cauchy sequences, Real Anal. Exchange, 30 (2004/2005), 123-128. 1

[8] H. Fast, Sur la convergence ststistique, Colloq. Math., 2 (1951), 241-244. 1

[9] A. R. Freedman, J. J. Sember, M. Raphael, Some Cesarò type summability spaces, Proc. London Math. Soc., 37 (1978), 508-520. 1

[10] J. A. Fridy, On statistical convergence, Analysis, 5 (1985), 301-313. 1, 2.2

[11] H. Gumuş, E. Savaş, On $S_{\lambda}^{L}(\mathrm{I})$-asymptotically statistical equivalent sequences, Proceedings of the International Conference on Numerical Analysis and Applied Mathematics (ICNAAM 2012), 2012 (2012), 936-941. 1

[12] U. Kadak, On weighted statistical convergence based on $(p, q)$-integers and related approximation theorems for functions of two variables, J. Math. Anal. Appl., 443 (2016), 752-764. 1

[13] U. Kadak, N. L. Braha, H. M. Srivastava, Statistical weighted B-summability and its applications to approximation theorems, Appl. Math. Comput., 302 (2017), 80-96. 1

[14] P. Kostyrko, W. Wilczynski, T. Salat, I-convergence, Real Anal. Exchange, 26 (2000), 669-686. 1, 2

[15] V. Kumar, A. Sharma, On asymptotically generalized statistical equivalent sequences via ideal, Tamkang J. Math., 43 (2012), 469-478. 1 
[16] B. K. Lahiri, P. Das, I- and I*-convergence in topological spaces, Math. Bohem., 130 (2005), 153-160. 1

[17] J. L. Li, Asymptotic equivalence of sequences and summability, Internat. J. Math. Math. Sci., 20 (1997), 749-758. 1

[18] M. Marouf, Asymptotic equivalence and summability, Internat. J. Math. Sci., 16 (1993), 755-762. 1, 2.1

[19] M. Mursaleen, $\lambda$-Statistical convergence, Math. Slovaca, 50 (2000), 111-115. 1, 1.1

[20] M. Mursaleen, A. Alotaibi, On J-convergence in random 2-normed spaces, Math. Slovaca, 61 (2011), 933-940. 1

[21] M. Mursaleen, V. Karakaya, M. Ertürk, F. Gürsoy, Weighted statistical convergence and its application to Korovkin type approximation theorem, Appl. Math. Comput., 218 (2012), 9132-9137. 1

[22] M. Mursaleen, S. A. Mohiuddine, On ideal convergence in probabilistic normed spaces, Math. Slovaca, 62 (2012), 49-62. 1

[23] M. Mursaleen, H. M. Srivastava, S. K. Sharma, Generalized statistically convergent sequences of fuzzy numbers, J. Intelligent Fuzzy Systems, 30 (2016), 1511-1518. 1

[24] F. Nuray, $\lambda$-Strongly summable and $\lambda$-statistically convergent functions, Iranian J. Sci. Tech. Trans. A Sci., 34 (2010), 335-339. 2.4, 2.5

[25] R. F. Patterson, On asymptotically statistically equivalent sequences, Demostratio Math., 6 (2003), 149-153. 1, $2,2.3$

[26] A. Sahiner, M. Gurdal, S. Saltan, H. Gunawan, Ideal convergence in 2-normed spaces, Taiwanese J. Math., 11 (2007), 1477-1484. 1

[27] T. Salat, On statistically convergent sequences of real numbers, Math. Slovaca, 30 (1980), 139-150. 1

[28] E. Savas, A-sequence spaces in 2-normed space defined by ideal convergence and an Orlicz function, Abstr. Appl. Anal., 2011 (2011), 9 pages. 1

[29] E. Savaş, Generalized summability methods of functions using ideals, Proceedings of the International Conference on Advancements in Mathematical Sciences (Antalya, Turkey), 2015 (2015), 5 pages. 1

[30] E. Savaş, On some new sequence spaces in 2-normed spaces using ideal convergence and an Orlicz function, J. Inequal. Appl., 2010 (2010), 8 pages. 1

[31] E. Savaş, On generalized statistical convergence in random 2-normed space, Iran. J. Sci. Technol. Trans. A Sci., 36 (2012), 417-423.

[32] E. Savaş, On J-asymptotically lacunary statistical equivalent sequences, Adv. Difference Equ., 2013 (2013), 7 pages. 1

[33] E. Savaş, On generalized statistically convergent function via ideals, Appl. Math. Inform. Sci., 10 (2016), 943-947. 1

[34] E. Savaş, $\Delta^{\mathrm{m}}$-Strongly summable sequences spaces in 2-normed spaces defined by ideal convergence and an Orlicz function, Appl. Math. Comput., 217 (2010), 271-276. 1

[35] R. Savaş, M. Basarir, ( $\sigma, \lambda)$-Asymptotically statistical equivalent sequences, Filomat, 20 (2006), 35-42. 1

[36] E. Savaş, P. Das, A generalized statistical convergence via ideals, Appl. Math. Lett., 24 (2011), 826-830. 1

[37] E. Savaş, H. Gumuş, A generalization on J-asymptotically lacunary statistical equivalent sequences, J. Inequal. Appl., 2013 (2013), 9 pages. 1

[38] I. J. Schoenberg, The integrability of certain functions and related summability methods, Amer. Math. Monthly, 66 (1959), 361-375. 1

[39] H. M. Srivastava, M. Et, Lacunary statistical convergence and strongly lacunary summable functions of order $\alpha$, Filomat, 31 (2017), 1573-1582. 1 